\section{Health care in Canada}

\section{by region}

I t's not just the numbers, it's what you do with them. This, says Glenda Yeates, president and CEO of the Canadian Institute for Health Information (CIHI), is the true value of the information in Health Care in Canada 2006, CIHI's 7 th annual report on the state of Canada's health system.

Data, produced with help from Statistics Canada, cover a 5-year period and a range of issues, from heart disease to regional variations in hysterectomy rates.

Key findings include:

- Women admitted with new heart attacks or strokes were more likely to die in hospital within 30 days than men (I6\% v. II\%).

- The 30-day in-hospital mortality rate for patients admitted with ischemic stroke changed little (1 $3 \%$ ) since I999-2000. Death rates for hemorrhagic stroke dropped from $37.7 \%$ to $32.8 \%$.

- After adjusting for population growth and aging, hospitalization rates for new heart attack patients fell about I9\% between I999-2000 and 2004-05. Rates for stroke hospitalizations in 2004-05 (I27 per Ioo ooo population) was about $23 \%$ lower for same period.

- After adjusting for age, gender and other illnesses, new heart-attack mortality rates within 30 days in some larger health regions (population over 75 ooo) were more than double those in others. Overall regional death rates ranged from $7.6 \%$ to $16.3 \%$.

- Regional rates for in-hospital deaths within 30 days of admission with a new stroke ranged from I $4.7 \%$ to $29.2 \%$. Of the 57 regions measured, I5 had rates statistically significantly higher than average; 7 were lower.

Yeates says the regional data hold great potential value for helping practitioners and politicians make improvements. And there are some surprises,

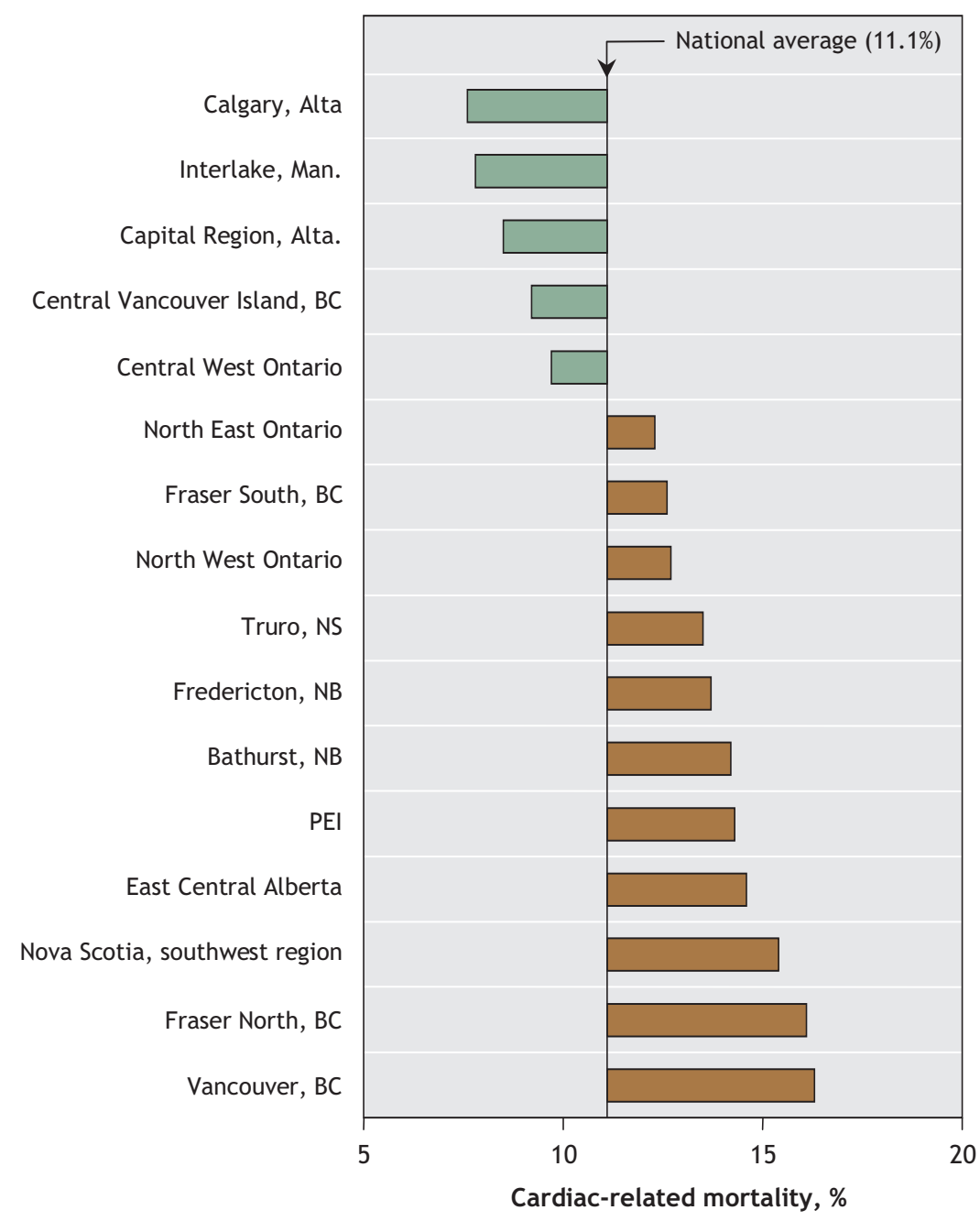

Fig. 1: Differences in heart mortality in regions with a population over 75 ooo. Source: Health Indiators 2006, CIHI

she says, including the fact that the data can be strikingly different within the same province and few predictions can be made based on data from a rural or urban setting (see Fig. I).

"What's heartening for us is that when we put out these indicators, we see regions really using them and digging down and trying to understand why their rate might be different than someone else's and how they can do better," Yeates told the CMAJ.

She points to the Ottawa region as an example. Unhappy with the 30-day in-hospital acute myocardial infarction (AMI) mortality rate (I4.I\% v. I2.6\% nationally) in the 2002 report, the Ottawa Heart Institute brought together hospital CEOs, paramedics and provin- cial officials to make treatment protocol changes. In 2003, the AMI mortality rates dropped to II.I\%.

"It isn't really about pitting one region against another," says Yeates. "It's about giving people the kind of information that they can then use and delve deeper to understand what does this mean for us."

Note: Due to historical differences in data collection, trend data exclude Newfoundland and Labrador (heart attack rates only), Quebec and British Columbia. Results for BC are available for the most recent years and are included in the regional comparisons. Pauline Comeau, Ottawa

DOI:I0.1503/cmaj.060748 